\title{
Predicted v. real prevalence of the 22q11.2 deletion syndrome in children with congenital heart disease presenting to Red Cross War Memorial Children's Hospital, South Africa: A prospective study
}

\author{
R de Decker, ${ }^{1}$ MSc, MB ChB, DCH, FCPaeds (SA), Cert Med Genet (Paeds); Z Bruwer, ${ }^{2,3}$ MSc, PhD; L Hendricks, ${ }^{1}$ MB ChB, DCH, FCPaed (SA); \\ M Schoeman, ${ }^{2,4}$ MSc; G Schutte, ${ }^{2,5}$ NDip, BTech; J Lawrenson, ${ }^{6}$ MB BCh, FCP (SA), MMed (Int) \\ Department of Paediatrics and Child Health, University of Cape Town and Red Cross War Memorial Children's Hospital, Cape Town, South Africa \\ ${ }^{2}$ Division of Human Genetics, Department of Pathology, Institute of Infectious Disease and Molecular Medicine, University of Cape Town, \\ South Africa \\ Genetic and Developmental Medicine Clinic, Sultan Qaboos University Hospital, Muscat, Sultanate of Oman \\ ${ }^{4}$ Division of Molecular Biology and Human Genetics, Stellenbosch University and Tygerberg Hospital, Cape Town, South Africa \\ Cytogenetics Laboratory, National Health Laboratory Service, Cape Town, South Africa \\ ${ }^{6}$ Department of Paediatrics and Child Health, Stellenbosch University and Tygerberg Children's Hospital, Cape Town, South Africa
}

Corresponding author: R de Decker (rik.dedecker@uct.ac.za)

Background. The 22q11.2 deletion syndrome (22qDS) has more than 180 associated phenotypic features, yet genotype-phenotype correlation remains obscure. Since many of the clinical characteristics are serious, yet treatable (including congenital heart disease), clinicians must maintain a high index of clinical suspicion to recognise a suite of co-occurring phenotypic features that suggest a diagnosis of $22 \mathrm{qDS}$. Óskarsdottir's scoring schedule (the 'O score') is generally used to suggest the need for confirmatory fluorescent in situ hybridisation (FISH) testing, using the TUPLE 1 probe. An O score of two or more indicates the need for FISH testing.

Objectives. A previous audit of FISH-positive results of patients with congenital heart disease at Red Cross War Memorial Children's Hospital (RCWMCH) revealed a clinical recognition rate of $1.7 \%$. However, we were concerned that the syndrome may be under-recognised in our setting. Our aims were therefore to assess the predictive value of ' $\mathrm{O}$ scoring' and to accurately determine the prevalence of 22qDS in our patient population.

Methods. A prospective trial of FISH testing every new patient with congenital heart disease presenting to RCWMCH was undertaken to accurately determine the prevalence of 22qDS. The results were then compared with the ability of the $\mathrm{O}$ score to indicate the need for FISH testing. Results. Testing of 125 patients detected deletions in six (4.8\%, 2.8 times the previously determined clinical detection rate), thereby vindicating our concern that 22qDS is under-diagnosed. Of these 125 patients, 37 had an O score of 2 or 3, yet only 6 were FISH-positive, giving the $\mathrm{O}$ score a positive predictive value of only $14 \%$.

Conclusion. Until a more robust alternative recognition tool is available, South African clinicians should use all clinical recognition criteria liberally to suggest the need for formal testing for 22qDS.

S Afr Med J 2016;106(6 Suppl 1):S82-S86. DOI:10.7196/SAMJ.2016.v106i6.11003

\section{Background}

Chromosome 22q11.2 deletion syndrome (22qDS) is the second most commonly occurring genetic syndrome (after trisomy 21) and is the most common microdeletion syndrome. The overall incidence is approximately 1 in 4000 live births. ${ }^{[1]}$ However, this figure might be an underestimate owing to the high rate of perinatal deaths secondary to severe congenital cardiac defects. ${ }^{[2,3]}$ Whilst the vast majority of probands have a de novo microdeletion of chromosome 22q11.2, about $10-15 \%$ of those affected inherit the 22q11.2 deletion from a parent with an autosomal dominant pattern of inheritance. Male and female genders are affected equally. ${ }^{[4,5]}$

This syndrome, with its broad range of phenotypic features (and previously known by many names), was at one stage thought to be different disease entities. Research has since confirmed a common underlying defect: a microdeletion of a segment (at 11.2) on the long arm (q) of chromosome $22^{[4-7]}$ - hence 22qDS.

The deletion contains numerous genes, but linkage analysis has identified a smaller, so-called critical region containing at least 25 30 candidate genes. This is known as the Di George critical region.
The typically deleted region (TDR) is usually $3 \mathrm{Mb}$ in size, which encompasses about 30 genes. These typical deletions occur in the majority of cases (90\%). ${ }^{[2,6]}$ However, smaller deletions of less than $1.5 \mathrm{Mb}$ have also been noted in $8 \%$ of cases, usually occurring distally to the TDR. The clinical severity of the syndrome has no correlation with the size of the deletion, and the reason for its wide phenotypic variability remains unclear. ${ }^{[2,5 \cdot 6]}$

As the most phenotypically diverse syndrome, more than 180 different abnormal features are associated with it. ${ }^{[8]}$ The clinical features with which affected individuals present can vary greatly and include congenital heart disease (CHD) in $80 \%$, ${ }^{[0]}$ palatal abnormalities, characteristic facial features, developmental delay and learning difficulties. As many as $77 \%$ of individuals suffer an immune deficiency regardless of their clinical presentation. Autoimmune disease is not infrequent, occurring in $10-20 \%$ of patients. ${ }^{[8]}$ Other common findings include hypocalcaemia, feeding problems, renal anomalies, hearing loss, laryngotracheo-oesophageal anomalies, seizures, skeletal abnormalities, neuropsychiatric disease and several malignancies. ${ }^{[3,4]}$ Owing to this diversity, a diagnosis in one field warrants careful 
investigation for other abnormalities, but only after confirmatory fluorescent in situ hybridisation (FISH) testing.

\section{Testing for $22 \mathrm{qDS}$}

The presence of the typical deletion of 22qDS is confirmed by FISH testing. The two probes commercially available for $22 \mathrm{q} 11.2$ FISH analysis are TUPLE1 and N25. The detection rate of FISH analysis using either probe is thought to be equivalent; however FISH using either one of these probes is not sensitive enough to detect smaller deletions $(<40 \mathrm{~kb})$ within the $22 \mathrm{q} 11.2$ region. ${ }^{[7]}$

Alternatives to FISH testing are now available, including multiplex ligationdependent probe amplification (MLPA), array comparative genomic hybridisation (CGH) and single nucleotide polymorphism (SNP) arrays. ${ }^{[1,13]}$ Although these methods are more expensive, they have advantages in terms of turnaround time and detection rates. $^{[12-15]}$

Owing to the broad spectrum of disease presentation in individuals with 22qDS, and because many clinical characteristics are treatable, it is imperative that clinicians maintain a high index of suspicion. In 2005 a study done in Sweden by Óskarsdottir et al. ${ }^{[14]}$ of 100 FISH-positive children and adolescents ascertained the most common clinical features associated with 22qDS. Based on this information, they developed a scoring schedule (here referred to as the ' $\mathrm{O}$ score'), suggesting that a score of more than 2 (out of a total of 8) implied the need for confirmatory FISH testing (Table 1).

Recognising 22qDS in South Africa Recognition of patients with 22qDS in our service may be difficult for two main reasons: the first is the difference in the facial dysmorphism of white and nonwhite patients confirmed to have 22qDS. While nearly all white and many non-white patients have distinctive facial dysmorphism to suggest the diagnosis, most of our non-white patients have extremely subtle facial dysmorphism of the syndrome, if any at all (Fig. 2). The second reason is that the phenotypic spectrum of 22qDS is extremely diverse ${ }^{[3]}$ including any of a set of 15 CHD diagnoses, ${ }^{[4]}$ of which, previously at our institution, only truncus arteriosus was mistakenly perceived to be indicative of a diagnosis of 22qDS. Consequently, we were concerned that (possibly owing to an institutional bias) the cohort of patients positively identified with 22qDS at the Red Cross War Memorial Children's Hospital (RCWMCH) under-represented its true prevalence.

Table 1. Diagnostic guidelines for consideration of genetic testing for 22qDS*

\begin{tabular}{|c|c|c|c|}
\hline & \multicolumn{3}{|c|}{ Age period } \\
\hline & Infant & Preschool & Childhood - adolescence \\
\hline 1 & Cardiac defect & Cardiac defect & Cardiac defect \\
\hline 2 & $\begin{array}{l}\text { Thymic hypoplasia } \\
\text { Immunodeficiency }\end{array}$ & Recurrent infections & $\begin{array}{l}\text { Recurrent infections } \\
\text { Autoimmunity }\end{array}$ \\
\hline 3 & Hypocalcaemia & Hypoparathyroidism & Hypoparathyroidism \\
\hline 4 & Feeding problems & - & - \\
\hline 5 & Cleft palate & Speech-language impairment & Velopharyngeal insufficiency \\
\hline 6 & - & $\begin{array}{l}\text { Developmental delay } \\
\text { Behavioural abnormalities }\end{array}$ & $\begin{array}{l}\text { Learning difficulties } \\
\text { Behavioural abnormalities }\end{array}$ \\
\hline 7 & $\begin{array}{l}\text { Any comorbidities, } \\
\text { e.g. anorectal } \\
\text { malformation }\end{array}$ & $\begin{array}{l}\text { Any comorbidities, } \\
\text { e.g. anorectal malformation }\end{array}$ & $\begin{array}{l}\text { Any comorbidities, } \\
\text { e.g. anorectal malformation }\end{array}$ \\
\hline 8 & $\begin{array}{l}\text { Subtle facial } \\
\text { dysmorphism }\end{array}$ & Subtle facial dysmorphism & Subtle facial dysmorphism \\
\hline
\end{tabular}

\section{Assessing the prevalence at} RCWMCH

The worldwide incidence of all CHD is said to be $8: 1000$, but it is known to be higher in developing countries, and an incidence of $1 \%$ seems more realistic. ${ }^{[1]}$ The population incidence of $22 \mathrm{qDS}$ is estimated to be 1:4000 newborns, ${ }^{[15]}$ therefore the fraction of 22qDS in a large random cohort of all newborn children with CHD should be around 1:50, or $2.0 \%$. Approximately $80 \%$ of children born with $22 \mathrm{qDS}$ will have $\mathrm{CHD},{ }^{[8,9]}$ so the prevalence of $22 \mathrm{qDS}$ in a random cohort of children with CHD at birth should be approximately $1.6 \%$.

In 2006, we audited our FISH-positive $22 \mathrm{qDS}$ results and found a clinical recognition rate of $1.7 \%$ (18 FISH-positive tests in 1048 patients that had been newly diagnosed with CHD (data presented at the SA Heart Annual Congress in 2006). However, for several reasons, to assess the real prevalence in any cohort it must be determined prospectively; that is what this study aimed to do.

\section{Objectives}

The present study was designed to determine accurately the prevalence of $22 \mathrm{qDS}$ in a cohort of children with CHD presenting to the cardiac services at RCWMCH. Furthermore, since we were using an international scoring system ${ }^{[12]}$ to suggest FISH testing, we wanted to test its predictive value in our patient population.

To determine the true prevalence of 22qDS in patients presenting to our cardiac service, we recruited patients prospectively and FISH tested all children with significant CHD.
Clearly, this approach will underestimate the birth incidence of 22qDS, since it does not take into account patients without CHD, as well as those who had died before presenting, or those who had not been clinically recognised and referred yet. The objective of the study, however, was to determine what our clinical pick-up rate should actually be and if it differed from our previously determined prevalence rate of $1.7 \%$.

\section{Methods}

A hospital-based prospective study was undertaken by recruiting all newly referred patients with a significant cardiac lesion presenting to the cardiology service at RCWMCH during a 7.5-month period. $\mathrm{RCWMCH}$ is the largest paediatric tertiary hospital in southern Africa and (together with the Tygerberg Children's Hospital) forms part of the Paediatric Cardiac Service of the Western Cape (PCSWC) of South Africa. All cardiac surgery and cardiac catheterisations in the PCSWC are done at RCWMCH. Patients with acquired heart disease, neonates with isolated patent ductus arteriosus or cases with identifiable cardiogenetic syndromes (e.g. Down syndrome) were excluded from the study.

Parents of the affected children were approached by a genetic counsellor and invited to participate in the research study for which written informed consent was obtained. In the event that a parent was not accessible, consent was also considered from the legal guardian. The hospital records of each enrolled patient were reviewed for notes or comments on dysmorphic features, extracardiac anomalies, or any other features 
suggestive of a syndromic cause (e.g. hypocalcaemia or thymic aplasia).

\section{O scoring}

Based on the diagnostic scoring guide for the consideration for genetic testing by Óskarsdottir et al. ${ }^{[14]}$ an assessment of the likelihood of a diagnosis of 22qDS was made in each patient. Each participant was given a score based on the presence of the number of clinical features suggestive of a 22qDS phenotype as classified in Table 1. The O score ranges from 1 to 8 depending on the number of clinical findings as suggested by the scoring system. The child's age (infant, preschool or childhood-adolescence) was considered during the scoring process, as prominent presenting features of the syndrome are known to illustrate age-related penetrance. An $\mathrm{O}$ score equal to or above 2 is taken as indicative of the need for formal FISH testing for the 22q11.2 deletion.

\section{2q11.2 deletion FISH testing}

The deletion status was determined by FISH testing using a fluorophorelabelled locus-specific identifier TUPLE1 probe, which hybridises to band q11.2 on chromosome 22. FISH analysis was performed on metaphase spreads from standard lymphocyte cultures. Analysis was performed according to the standardised procedure of the local diagnostic laboratory (National Health Laboratory Service, Cytogenetics Laboratory). To avoid additional venipuncture, where possible, blood for FISH analysis was drawn at the time of surgical or cardiac catheterisation procedures or when invasive sampling was required for routine work-up.

\section{Ethical considerations}

This study was approved by the Human Ethics Research Committee (HREC) of the Faculty of Health Sciences, University of Cape Town (UCT HREC no.: 204/2002). All patients who tested positive for $22 \mathrm{qDS}$ were referred for formal genetic counselling and managed in collaboration with a clinical geneticist.

\section{Results}

Five hundred and eighty newly referred children with CHD were seen at the cardiology service at RCWMCH during the study period (Fig. 1). Of these, 191 patients met the inclusion criteria but for various reasons, including logistical and administrative (such as delayed return to RCWMCH from rural referral centres), only 150 patients could be enrolled into the study. Written informed consent was obtained subsequent to enrolment for 142 patients. None of the

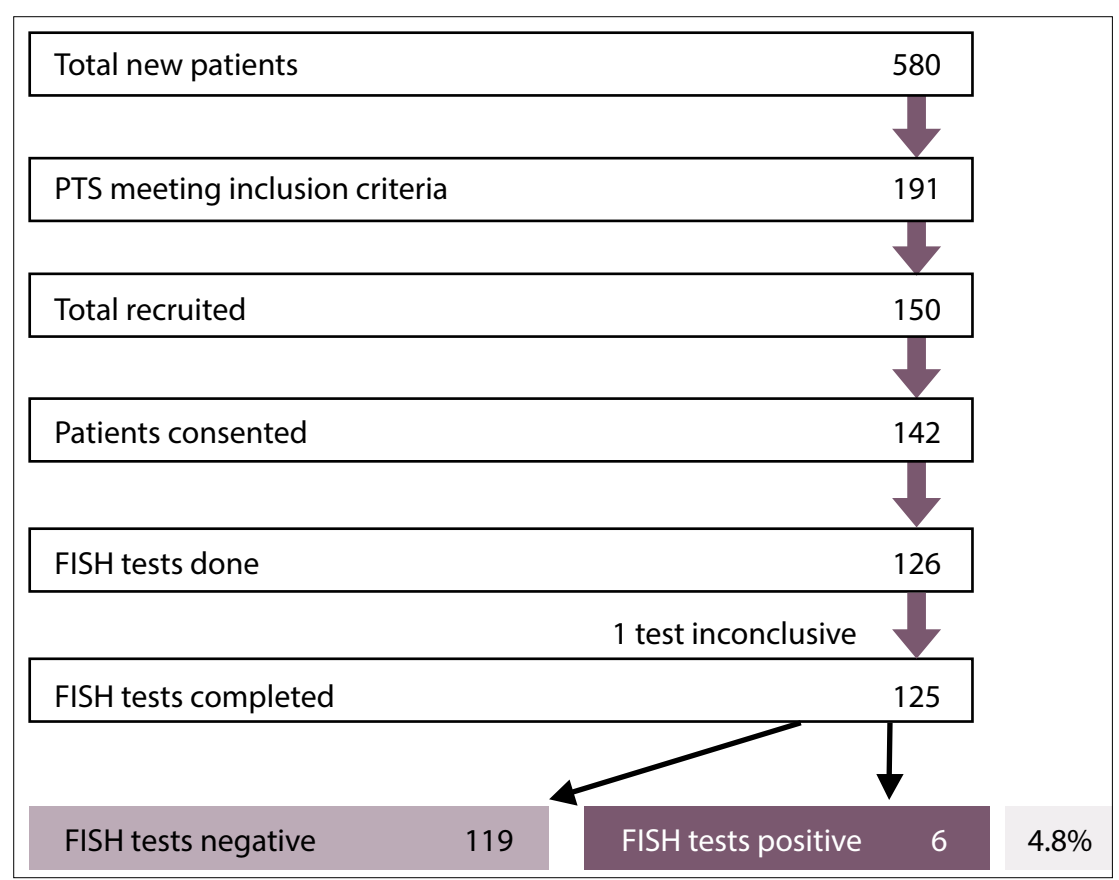

Fig. 1. Summary of recruitment and enrolment of the 125 patients FISH tested for the 22qDS, with their results. The reasons for the marked attrition between patients meeting the inclusion criteria and those FISH tested are given in the text.

parents of the studied individuals reported a familial occurrence or positive family history.

Four patients died before a blood sample could be collected, the parents of two patients declined involvement in the study and for two patients, no parent or legal guardian could be identified to provide informed consent. A further 16 patients were lost to follow-up. Therefore only 125 patients were available for FISH testing. Based on our initial population estimate (of $1.7 \%$ ) we predicted 2 - 3 FISH-positive results.

Distribution of scores based on diagnostic guidelines

One hundred and twenty-five patients were $\mathrm{O}$ scored according to the clinical diagnostic guidelines provided in Table 1. The patients' congenital cardiac anomaly was diagnosed by echocardiography and the specific lesion of each FISH-positive patient is summarised in Table 2. Dysmorphic features and extracardiac anomalies were based on documented findings from an evaluation performed by either a cardiologist or clinical geneticist.

The results of the $\mathrm{O}$ scoring are summarised in Table 3. Since all participants had CHD, all 125 patients had a baseline O score of 1 . Eightytwo patients had an isolated cardiovascular anomaly (score of 1 ), whereas 30 patients were found to have a cardiac anomaly plus one clinical feature in one of the remaining seven domains (score of 2), and 15 patients had a
Table 2. Cardiac lesions of the six 22qDS

\begin{tabular}{ll}
\hline Diagnosis & $\boldsymbol{n}$ \\
\hline Ventricular septal defect & 2 \\
$\begin{array}{l}\text { Tricuspid atresia and interrupted } \\
\text { aortic arch }\end{array}$ & 1 \\
$\begin{array}{l}\text { Tetralogy of Fallot with left aortic arch } \\
\text { Tricuspid atresia with right aortic arch }\end{array}$ & 1 \\
$\begin{array}{l}\text { Ventricular septal defect with right } \\
\text { aortic arch }\end{array}$ & 1 \\
Total & 6
\end{tabular}

Table 3. Distribution of $\mathrm{O}$ scores among FISH-positive and FISH-negative patients. An $\mathrm{O}$ score of 2 or more suggests the need for FISH testing FISH results

O score Negative Positive Totals

$\begin{array}{llll}1 & 82 & 0 & 82\end{array}$

$\begin{array}{llll}2 & 28 & 3 & 31\end{array}$

$\begin{array}{llll}3 & 9 & 3 & 12\end{array}$

$\begin{array}{llll}\text { Total } & 119 & 6 & 125\end{array}$

cardiac malformation and clinical features of two of the seven domains (score of 3). No patient had an $\mathrm{O}$ score of more than 3.

\section{Deletion frequency}

22qDS FISH testing results were available for 125 samples of the 126 tested patients. (FISH-positive) patients 


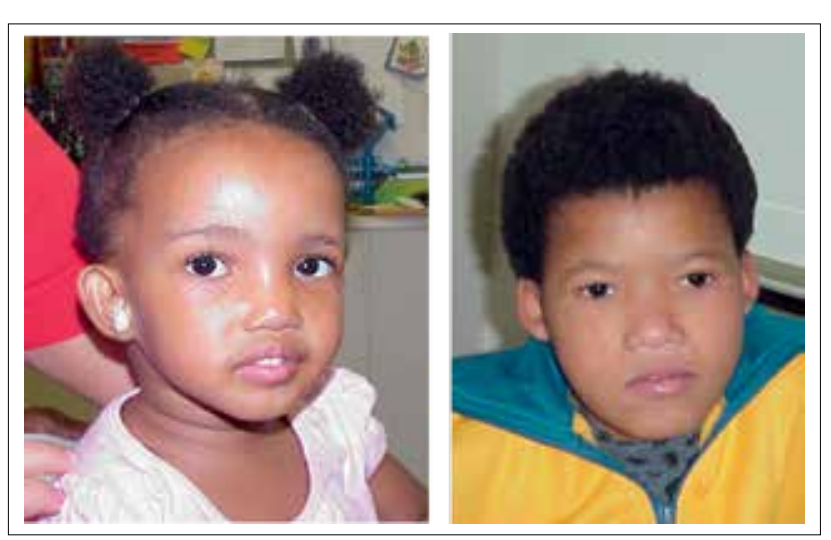

Fig. 2. Subtle 22qDS facial dysmorphism typical of our non-white patient population. Patient images provided with the consent of their parents or legal guardians.

The result of one patient was technically inconclusive. Deletions at $22 \mathrm{q} 11.2$ were detected in 6 of the remaining 125 patients (4.8\% FISHpositive: 2.8 times the previous rate of $1.7 \%$ ). Deletions were seen in patients with one of three cardiac defects including tricuspid atresia and interrupted aortic arch, tricuspid atresia with right aortic arch, a ventricular septal defect with right aortic arch, an isolated ventricular septal defect, and a tetralogy of Fallot with a left aortic arch (Table 2).

No FISH-positive cases were identified in the group of patients with an $\mathrm{O}$ score of $1,6.7 \%$ of cases were found to be FISH positive in the group scoring 2 and $30.8 \%$ in the group within scoring category 3 (Table 3). The mean score for FISH-positive cases was 2.7 and for FISH-negative cases 1.4, thereby justifying the FISH testing criterion of an $\mathrm{O}$ score of more than 2.

However, 28 patients and 9 patients with scores of 2 and 3, respectively, were FISH negative. In other words, of the 43 patients who scored more than 2 (thereby indicating that FISH testing is warranted), only six were FISH positive. This gave the $\mathrm{O}$ scoring system a positive predictive value of only $15.9 \%$, but a sensitivity of $100 \%$, meaning that none of the patients with an O score of less than 2 were FISH positive.

There remains the small possibility that patients with $\mathrm{O}$ scores $>1$, but non-FISH positive, are indeed deleted, due to the presence of a distal deletion, ${ }^{[16]}$ or a smaller deletion that does not contain the TUPLE1 gene, or too small to be detected $(<40 \mathrm{~kb})$. This speculation may be resolved with the use of the more sensitive screening probes, such as MLPA or SNPs.

\section{Discussion}

This study was motivated by a concern that in our cardiac service, $22 \mathrm{qDS}$ is clinically underdiagnosed, and this concern has been vindicated. Prospective FISH testing of all children with significant CHD has found a FISH-positive rate for 22qDS of $4.8 \%$ (6 patients) in 125 newly referred patients. This is 2.8 times the clinically detected rate of $1.7 \%$ found retrospectively in 2006 . We have therefore shown that 22qDS had been clinically under-recognised by almost threefold in patients presenting to our cardiac service. Speculation around the reasons for this is difficult, but the lack of the characteristic facial features of 22qDS in our patients may be contributory.

The Óskarsdottir scoring system was developed in Sweden. ${ }^{[14]}$ In contrast, our patient population is predominantly non-white, nearly always lacking the obvious dysmorphic facial features that we have come to associate with white FISH-positive patients. We are currently unsure if this phenotypic variation extends to the other typically described phenotypic characteristics of 22qDS, and a review is currently underway of all our FISH-positive patients to assess this. The cardiac lesions in this cohort of FISH-positive patients (Table 2) are all known to occur in 22qDS, but the number is too small to draw firm conclusions from these limited findings to assist in developing changes to clinical recognition models. It is worth noting, however, that two patients had isolated ventricular septal defects (VSDs), which are not conotruncal lesions. Had FISH testing been performed only on patients with conotruncal lesions, these two cases would not have been detected.

Óskarsdottir et al ${ }^{[14]}$ suggested that a score of 2 or more indicates the need for formal FISH testing. O scoring of our cohort had a positive predictive value of only $14 \%$, emphasising the difficulty in clinical recognition of our patients with this syndrome. Whereas the international scoring schedule for the 22qDS is poorly predictive for FISH testing in the southern African context, it is highly sensitive in suggesting those that do not require testing: an $\mathrm{O}$ score of 1 always predicted a negative FISH test correctly. Therefore the $\mathrm{O}$ score is of limited aid in the diagnosis of the syndrome in our local population, but remains a robust tool to suggest refraining from testing those with too low an $\mathrm{O}$ score.

The need for a firm diagnosis is important since it carries several serious implications ${ }^{[17]}$ including, inter alia, cascade screening of parents and family for the deletion, a focused search for known comorbidities, the need for irradiated blood for cardiopulmonary bypass or blood transfusion, assessment for immunodeficiency and hypocalcaemia, and counselling with regard to potential future problems, such as learning difficulties and schizophrenia. ${ }^{[17]}$ It is therefore important that a high index of suspicion is maintained for this syndrome. Until a local recognition tool is developed, clinicians should apply all recognition criteria liberally to suggest the need for FISH testing, particularly in non-white patients. To reduce the cost of too many needless FISH tests, we have to define the cardinal phenotypic features of 22qDS in our patient population more clearly; such a study is currently underway.

\section{Study limitations}

The study suffered from several logistical problems, mostly related to the nature of clinical healthcare delivery in a resource-constrained environment, including significant loss to follow-up and referral from distant rural healthcare centres limiting participation in the study. Although several attempts were made to enroll missed patients if a follow-up visit occurred during the study period, 41 cases were missed as a result of the genetic counsellor being unable to approach the family before the patient was discharged from the hospital. No FISH-positive white children were detected in this cohort.

\section{Conclusions}

If patients in a South African setting are selected for FISH testing for the 22qDS on clinical suspicion only, the disorder will be severely under-diagnosed. We have shown by prospective testing that the prevalence of the disorder may be nearly three times higher than clinically suspected in a population with $\mathrm{CHD}$ referred to the PCSWC tertiary cardiology unit. This may be owing to the fact that the international clinical scoring system currently in use may not be applicable to our patient population. The reasons for this are not clear, but are currently under investigation.

Acknowledgements. We thank the team members of the cardiology and genetic services at the RCWMCH and the cytogenetics NHLS laboratory at Groote Schuur Hospital for their unfailing support. The reviewers of this article made several valuable suggestions for improvement of the draft. 


\section{References}

1. Hoffman JI, Kaplan S. The incidence of congenital heart disease. J Am Coll Cardiol 2002;39(12):18901900.

2. McDonald-McGinn DM, Driscoll DA, Emanuel BS, et al. Detection of a 22q11.2 deletion in cardiac patients suggests a risk for velopharyngeal incompetence. Pediatrics 1997;99(5):E9.

3. Kitsiou-Tzeli S, Kolialexi A, Fryssira H, et al. Detection of 22q11.2 deletion among 159 patients with Di George/Velocardiofacial syndrome features. In Vivo 2004;18(5):603-608.

4. McDonald-McGinn DM, Emanuel BS, Zackai EH. GeneReviews. 22q11.2 Deletion Syndrome. http:// www.ncbi.nlm.nih.gov/books/NBK1523/ (accessed 15 September 2015)

5. Yamagishi $H$. The 22q11.2 deletion syndrome. Keio J Med 2002;51(2):77-88

6. De Decker HP L 2001:3(1):2-5. DOI:10.109700125817-200101000-00002

7. Webbe $\mathrm{SA}$, Hach 7. Webber SA, Hatchwell E, Barber JC, et al. Importance of microdeletions of chromosomal region 22q11 as a cause of selected malformations of the ventricular outflow tracts and aortic arch: A three-year

prospective study. J Pediatr 1996;129(1):26-32.
8. Digilio MC, Marino B, Capolino R, Dallapiccola B. Clinical manifestations of deletion 22q11.2 syndrome (DiGeorge/velo-cardio-facial syndrome). Images Paediatr Cardiol 2005;7(2):23-34.

9. Hacihamdioğlu B, Hacihamdioğlu D, Delil K. 22q11 deletion syndrome: Current perspective. Appl Clin Genet 2015;8:123-132. DOI:10.2147/TACG.S82105
10. Bassett AS, McDonald-McGinn DM, Devriendt K, et al. Practical guidelines for managing patients with 22q11.2 deletion syndrome. J Pediatr 2012;159(2):332-339. DOI:10.1016/j.jpeds.2011.02.039

11. Emanuel BS. Molecular mechanisms and diagnosis of chromosome 22q11.2 rearrangements. Dev Disabil Res Rev 2008;14(1):11-18. DOI:10.1002/ddrr.3

12. Michaelovsky E, Frisch A, Carmel M, et al. Genotype-phenotype correlation in 22q11.2 deletion syndrome. BMC Med Genet 2012;13:122. DOI:10.1186/1471-2350-13-122

13. Hu Y, Zhu X, Yang Y, et al. Incidences of micro-deletion/duplication 22q11.2 detected by multiplex ligation-dependent probe amplification in patients with congenital cardiac disease who are scheduled for cardiac surgery. Cardiol Young 2009;19(2):179-184. DOI:10.1017/S1047951109003667

14. Óskarsdottir S, Persson C, Eriksson BO, Fasth A. Presenting phenotype in 100 children with the 22q11 deletion syndrome. Eur J Pediatr 2005;164(3):146-153. DOI:10.1007/s00431-004-1577-8

15. McDonald-McGinn DM, Sullivan KE. Chromosome 22q11.2 deletion syndrome (DiGeorge syndrome/velocardiofacial syndrome). Medicine (Baltimore) 2011:90(1):1-18, DOI:10.1097/ MD.0b013e3182060469

16. Burnside RD. 22q11.21 deletion syndromes: A review of proximal, central, and distal deletions and their associated features. Cytogenet Genome Res 2015;146(2):89-99. DOI:10.1159/000438708

17. Kobrynski LJ, Sullivan KE. Velocardiofacial syndrome, DiGeorge syndrome: The chromosome 22q11.2 deletion syndromes. Lancet 2007;370(9596):1443-1452. DOI:10.1016/S0140-6736(07)61601-8 